\title{
How old are young people when they start having sex? Unravelling the applicability of Cox proportional hazards regression
}

\author{
Hanneke de Graaf $^{1}$ (D) Judith ter Schure ${ }^{2}$ (D) Geneviève A. F. S. van Liere ${ }^{3}$ \\ Received: 11 May 2020 / Accepted: 11 June 2021 \\ (C) The Author(s), under exclusive licence to Springer-Verlag GmbH Germany, part of Springer Nature 2021
}

\begin{abstract}
Purpose How old are young people when they start having sex? Although this question is important for educators and policymakers, its answer can easily mislead due to methodology that does not take into account age-censoring and generational trends. This study investigated whether 1-year birth-year cohorts can be jointly modeled by Cox proportional hazards regression to estimate sexual debut ages and to investigate age trends for different sexual behaviors.

Method We used pooled data from three Dutch periodic population-based surveys, for which 33,377 participants, born between 1980 and 2004, completed an online questionnaire.

Results Cox proportional hazards regression is appropriate if hazard changes over birth years appear proportionally similar among ages. The Dutch data shows such hazard changes for petting, oral sex, and sexual intercourse, but not for masturbation, French kissing, or manual sex. For sexual intercourse, the Cox model estimates can be interpreted as an approximate linear increase of 1 month in the median sexual debut age per birth-year cohort, resulting in an estimated debut age of 18.17 years in the current (2020) generation of Dutch adolescents.

Conclusion If the assumptions are met, we recommend using the Cox regression modeling approach to estimate how old young people are when they start having sex, since this method yields precise and current debut ages by pooling information across birth-year cohorts without arbitrarily combining them in multiple-year groups. The age of first intercourse is increasing, so continuous sexuality education throughout high school is advisable, in addition to early sexuality education.
\end{abstract}

Keywords Population study $\cdot$ Sexual debut $\cdot$ Cox regression $\cdot$ Adolescence

\section{Introduction}

The timing of first sexual intercourse is a topic of interest in many publications (Buhi and Goodson 2007). Researchers have shown that an early sexual debut correlates with a varying array of adverse outcomes, such as the risk of unplanned pregnancy, sexually transmitted infections, dating violence, and less psychosocial well-being (e.g., depression, substance use, and low self-esteem) (Epstein et al. 2018; Golden et al.

Hanneke de Graaf

h.degraaf@ rutgers.nl

1 Research Department, Rutgers, P.O. Box 9022, 3506 GA Utrecht, The Netherlands

2 Significant Help, Statistical Consultancy, Utrecht, The Netherlands

3 Public Health Service South Limburg, Geleen, The Netherlands
2016; Ihongbe et al. 2017; Lara and Abda 2016; Osorio et al. 2017; Sandfort et al. 2008; Vasilenko et al. 2016). In light of these physical and psychological health risks, an abundance of research focuses on correlates of early sexual debut (e.g., Lara and Abda 2016; Young et al. 2018).

These studies in general do not answer the question, "How old are young people when they start having sex?" This is an important question for educators and policymakers to guide the choice of topics to address in sexuality education at different ages (BZgA 2010). This question is also of interest for young people themselves, as age norms for sexual initiation influence their sexual behavior (Madkour et al. 2014). In addition, because of the link to sexual health outcomes, educators and policymakers are always keen to monitor increases or decreases in the age of first sexual intercourse.

Although this question is thus of great interest to educators, policymakers, and young people themselves, there is no simple way to answer it. Studies on patterns of sexual initiation or on the causes or consequences of an early sexual debut often 
present the conditional mean ages of first sexual experiences (i.e., the mean age in the group for whom the first sexual experience had already happened) (Cha et al. 2016; Epstein et al. 2018; Tornello et al. 2014; Halpern and Haydon 2012; Haydon et al. 2012), conditional median ages (i.e., the midpoint of the frequency distribution of the ages at which sexually experienced adolescents had sex for the first time) (Liu et al. 2015), or conditional quartiles (Holway 2015). Apart from their conditional nature (i.e., the exclusion of sexually inexperienced people), these estimates are often based on right-censored data, meaning that they include participants, and therefore experiences, up to a certain age.

Since only data of sexually experienced participants are included in conditional means, these estimates are not generalizable to a straightforward general population. Also, in younger samples, the right-censoring causes consistent downward bias, because these estimates exclude sexual experiences after the upper age limit of the sample. Furthermore, these parameters are often misunderstood as the mean or median age of the whole sample or population. Earlier research showed that if students are presented with a conditional mean age of first sex, they are more likely to overestimate the amount of sexual initiation among their peers (De Irala et al. 2014). Many studies on the correlates of or trends in sexual initiation ages present the proportion of sexually active adolescents within a young sample, for example 12-16-year-olds (e.g., Ethier et al. 2018; Ihongbe et al. 2017; Neville et al. 2017; Osorio et al. 2017; Ramiro et al. 2015). Although this percentage is less sensitive to misinterpretation, it does not shed light on the age of first sexual experience.

The most reliable estimates of the age of first sex combine status data (i.e., the proportion of sexually active participants in every age group) with data on the recalled age of first sex among the sexually active participants (Zaba et al. 2002, 2004). Survival analysis is a statistical method that achieves this, herewith used to estimate unconditional mean and median ages. However, survival analysis can only be applied to a sample with a wide age range if there are no changes over time. If one expects or examines trends over time, the analyses should be conducted separately for birth-year cohorts. Older birth-year cohorts - with respondents who had their first experiences a long time ago - do not give insight into current sexual debut ages. A few studies presented unconditional median ages in different cohorts using survival analyses, based on data from the British National Survey of Sexual Attitudes and Lifestyles (Lewis et al. 2017; Mercer et al. 2013), Youth Risk Behavior Surveillance System (Cavazos-Rehg et al. 2009), National Survey of Family Growth (Finer 2007), and Demographic and Health Surveys Program (Zaba et al. 2004).

Most of these studies use 5- or 10-year cohorts and give insight into trends in the age of sexual initiation and current median ages. However, the arbitrary use of 5-year cohorts could inflate or mask trends, and yields less precise estimates of sexual debut ages. The use of 1-year age groups has other limitations, especially the large sampling errors in the (small) age groups. In the present study, we assess whether 1-year birth-year cohorts can be jointly modeled by Cox proportional hazards regression to estimate current and accurate sexual debut ages. In addition, we will examine whether this modeling approach is applicable to first sexual experiences other than sexual intercourse (e.g., first kiss, first petting), because these are important developmental milestones as well (De Graaf et al. 2009). If not, we will explore why this is the case. If applicable, we will use this method to estimate the ages of first sexual experiences in the current generation of Dutch adolescents more precisely and to investigate trends in these ages.

\section{Methods}

\section{Sample}

We used pooled data from "Sex under the age of 25," a periodic population-based survey of the sexual health of young people aged 12-24 in the Netherlands. In 2004, 2011, and 2016, participants were recruited in two ways. Younger participants (aged 12-16) were recruited via randomly selected schools and older participants (aged 17-24) via a sample drawn from the municipal population registers. Weighting techniques were applied (by sex, age, educational level, and ethnic background) to ensure that the samples were representative of the Dutch youth population at each year of measurement. The procedures for data collection and the composition of the different samples were respectively described in De Graaf et al. (2009, 2015, 2018). Participants born between 1980 and 2004 were selected from the pooled data set, because of sufficient sample sizes. The final sample consisted of 33,377 participants. Table 1 shows the demographic characteristics of the weighted sample. The study protocols were

Table 1 Demographic characteristics of the sample (weighted \%)

\begin{tabular}{lll}
\hline & Boys & Girls \\
\hline Birth year & & \\
$1980-1984$ & 5.5 & 5.4 \\
$1985-1989$ & 11.9 & 12.5 \\
$1990-1994$ & 26.5 & 26.4 \\
$1995-1999$ & 32.8 & 32.2 \\
$2000-2004$ & 23.3 & 23.5 \\
Ethnicity & & \\
Dutch or Western & 84.5 & 84.6 \\
Non-Western & 15.5 & 15.4 \\
Total & 50.3 & 49.7 \\
Unweighted N & 13,754 & 19,644 \\
\hline
\end{tabular}


exempt from formal medical-ethical approval under Dutch law.

\section{Measures}

The participants completed an online questionnaire including demographics and sexual health measures. The following measures were included in the current study:

Birth year. Birth year was calculated by subtracting the age during survey completion from the date of survey completion. In 2005 and 2011, age during survey completion was measured in years and months. In 2016 this was measured in years for privacy reasons.

Timing of first sexual experiences. We assessed the timing of six forms of first sexual experiences: masturbation, French kissing, petting, oral sex (i.e., oral stimulation of the genitals), manual sex (i.e., manual stimulation of the genitals), and sexual intercourse (i.e., penile-vaginal). For each form of sexual behavior, participants were first asked whether or not they had experienced this form of sexual behavior (= current status data). If they answered "yes," they were asked at what age they had this experience for the first time. These recalled ages were reported in years (ranging from before age 8 through age 24). With the exception of masturbation, all recalled ages were left-censored at age 12 . Due to the age range of the sample, recalled ages could not be older than age 24 . Current status data and recalled ages were combined in the variable "timing." If the participant had experience of a form of sexual behavior, "timing" was the recalled age of their first experiences with this behavior. For inexperienced participants, "timing" was right-censored at their age during survey completion.

\section{Statistical analyses}

We assessed whether 1-year birth-year cohorts could be modeled jointly by Cox regression, with a Kaplan-Meier baseline survival curve, including "birth year" as a covariate. This model estimates a log-linear trend relating ratios of hazards to birth-year cohorts, indicating a proportional change in hazard per later birth year that is the same at each age. An estimated hazard for a specific age and birth-year cohort is the probability that a participant born in this year who has not had a first experience yet will do so in the next year. This method is able to relate estimated survival in previous and successive birth years and to pool information from all birth-year cohorts to estimate the baseline Kaplan-Meier survival curve, resulting in smaller sampling errors compared to estimates for individual birth-year cohorts. The Cox regression modeling approach depends substantially on the proportional hazards assumption, which is therefore of main interest in the assessment of the applicability of the model. This proportional hazards assumption means that, for example, if an inexperienced 16-year old born in 1994 has a lower probability of having a first sexual experience in the next year than a 16-year old born in 1993, the model assumes the same percentage change in hazard for any other age. Ergo, the occurrence of a "waterbed effect" (i.e., that the chances in one age group have decreased because they have increased in subsequent age groups) would not comply with the proportional hazards assumption. This assumption seems plausible for the timing of sexual experiences if the cultural changes that affect our event estimates are gradual and do not influence young people of different ages differently.

The proportional hazards test was used to assess the Cox model fit. We used IBM SPSS Complex Samples to account for design effects, because of oversampling in some regions and weighted data. The proportional hazards test rejects the proportional hazards hypothesis if the model fit is considerably worse than that of an alternative model that includes time-dependent predictors. Exponentiated estimates $[\operatorname{Exp}(B)]$ are reported for those sexual experiences that fit the Cox model.

In this paper we base the median ages of various sexual experiences (i.e., the age for which $50 \%$ of each birth-year cohort has had this experience) on the survival curves that were estimated by the Cox model. We approximated the median age by a linear interpolation between the single-year age group where the $50 \%$ survival had not been reached and the single-year age group where this boundary had been crossed. Our data set did not include the respondent's age at a monthly precision, but only age in years at first event and birth year. Therefore, to obtain the median age, the ages were increased by 0.5 year, since participants who report to have had a first experience at a certain age (e.g., at age 16) had this experience on average 6 months later (e.g., at age 16.5).

\section{Results}

For petting, sexual intercourse, and oral sex, the proportional hazards assumption tests were nonsignificant, indicating that the proportional hazards assumption could not be rejected. For these forms of sexual behavior, changes in birth year significantly predicted changes in survival. Exponentiated estimates ranged from .970 to .975 , indicating that a participant in a specific birth year who has not had this sexual experience has a 3.0 to $2.5 \%$ lower probability of doing so in the next year than a participant of the same age from an earlier birthyear cohort. For these experiences, the shift in median age from one birth-year cohort to the next was almost linear (as shown in Fig. 1), with an almost invisible bend upwards at the beginning and end of the curve. Such a linear approximation is 
Fig. 1 Linear trends in median ages of first petting, sexual intercourse, and oral sex

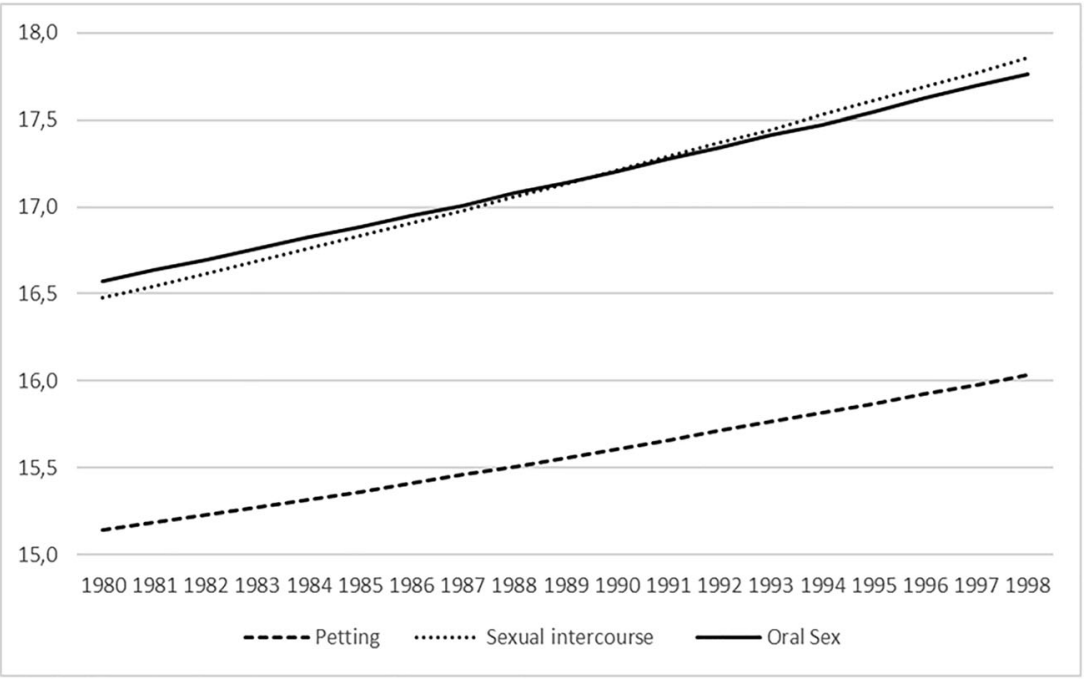

only fairly accurate if the hazard changes by birth year are small percentages $[\operatorname{Exp}(B)$ close to 1] and the range of birth years is not too large. For $\operatorname{Exp}(B)=0.970$ (intercourse) and the current birth year of interest 2002, the resulting approximation error in the median is smaller than half a month, for which details are given by Ter Schure (2020). We consider this error to be negligible and therefore choose to communicate the shift in median age for successive birth years as a linear change and to use that linear change to extrapolate medians for birth years in which the majority of the respondents still report being inexperienced (cohorts 1999-2004, therefore not shown in Fig. 1).

These results enable us to estimate rather precise debut ages of petting, oral sex, and sexual intercourse for the current generation. Young people have their first experiences of petting, oral sex, and sexual intercourse at successively later ages, which is illustrated in Fig. 1. For the current generation of Dutch youth, the median age can be obtained from the estimated trends in hazards using Table 2, by subtracting 1995 (the reference year) from the birth year, multiplying the result by the average shift per year, and adding the result of the latter to the median age in 1995. For example, the median age for first sexual intercourse was 17.61 for participants born in 1995 and the average shift per year in median age is 0.08 . Ergo, $50 \%$ of the participants born in 2003 will have experienced sexual intercourse when they are 18.25 years old (i.e., $17.61+$ $[(2003-1995) \times 0.08])$.

For masturbation, French kissing, and manual sex, the proportional hazards assumption tests were significant, showing that for these sexual experiences, the estimated percentage change in the hazard for two successive birthyear cohorts is not the same for all age groups. To gain more insight into this problem, we plotted the hazards for a selection of birth-year cohorts that were obtained by estimating a Kaplan-Meier survival curve separately for each birth-year cohort (Fig. 2). The plots do not show indications of a log-quadratic model, since there is neither a decrease in proportional hazards for the older cohorts nor an accelerated increase in proportional hazards for the younger cohorts. However, the plots show several "waterbed effects." The probability that a participant born in 1989 who has not yet French kissed will do so in the next year is the highest at age 16. For the 1990 birth-year cohort, this probability has deceased for age 16, in favor of age 15 and age 18. For the 1992 birth-year cohort, the highest probability has shifted from age 18 to age 19 .

Table 2 Parameter estimates for analyses with birth year as a covariate

\begin{tabular}{lllllll}
\hline & Exp(B) & $95 \%$ CI & $p$ & $\begin{array}{l}\text { Test of proportional hazards } \\
(p)\end{array}$ & $\begin{array}{l}\text { Median age for birth-year } \\
1995^{\mathrm{a}}\end{array}$ & $\begin{array}{l}\text { Approximate shift per birth year in median } \\
\text { age }\end{array}$ \\
\hline Masturbation & - & - & - & .000 & - & - \\
French kissing & - & - & - & .003 & - & - \\
Petting & .974 & $.971-.977$ & .000 & .466 & 15.87 & 0.05 \\
Manual sex & - & - & - & .037 & - & - \\
Intercourse & .970 & $.967-.974$ & .000 & .895 & 17.61 & 0.08 \\
Oral sex & .975 & $.971-.978$ & .000 & .527 & 17.55 & 0.07 \\
\hline
\end{tabular}

${ }^{\mathrm{a}}$ The reference year is 1994.9 
Fig. 2 Hazards for age of first French kissing in four birth-year cohorts

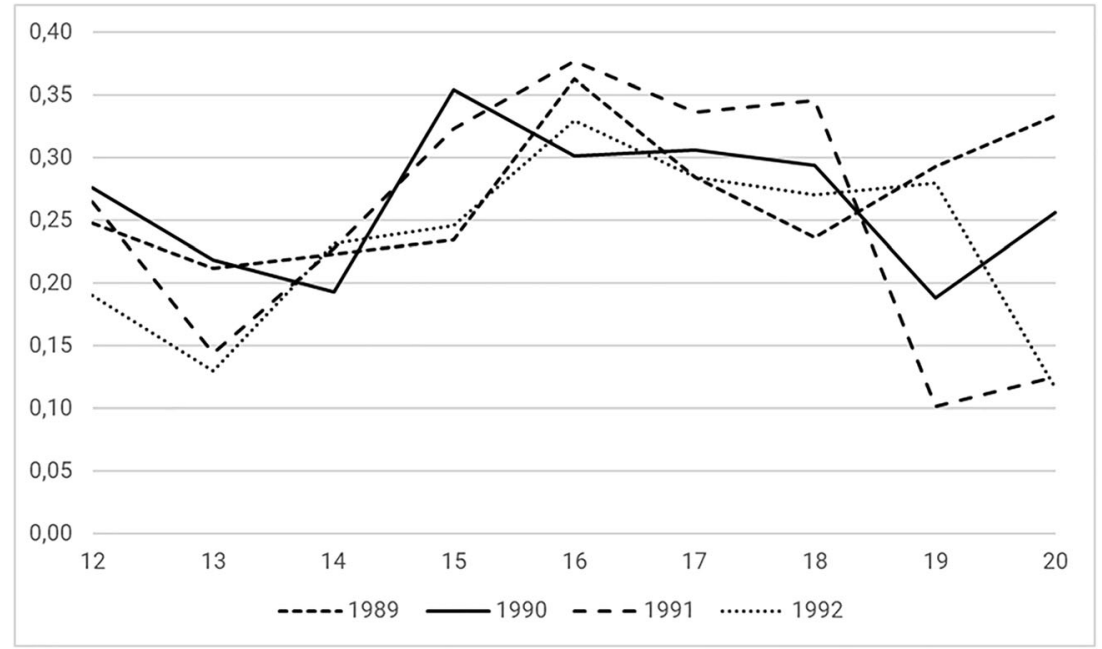

Searching for more complicated relations between hazards and birth-year cohorts comes at the risk of overfitting the data. For sexual behaviors where the proportional hazards assumption tests are significant, we therefore suggest estimating survival and median ages separately for each birth-year cohort. It has to be noted that the sampling errors will be larger due to the small 1-year cohort samples. The median ages for each single birth-year cohort for masturbation, French kissing, and manual sex resulting from these analyses on our data are shown in Fig. 3.

\section{Discussion}

In the present study, we assessed whether sexual debut ages can be modeled by Cox proportional hazards regression and whether the applicability of this method varies between first experiences (e.g., first French kiss, first sexual intercourse). The results showed that this model can be used effectively for modeling the ages of first experience of petting, oral sex, and sexual intercourse. However, the Cox regression modeling approach was not applicable for French kissing, masturbation or manual sex. Closer inspection of the hazard plots showed several "waterbed effects," indicating that in some birth-year cohorts, the hazard for starting French kissing, masturbation, or manual sex suddenly shifted to an earlier or later age, at the expense of the hazards at other ages. We cannot explain why these "waterbed effects" occur for these sexual behaviors. It is possible that these debut ages are less affected by gradual societal changes (e.g., changes in social media usage, parenting or economy) that underlie the linear trends in the other sexual debut ages, and more by temporary factors or chance.

The applicability of the Cox proportional hazards regression for petting, oral sex, and sexual intercourse enables us to estimate current debut ages for these forms of sexual behavior rather precisely. Based on the trends reported among their peers at younger ages, half of the participants born in 2003 (who will turn 18 in 2021), for example, will have their first experience of oral sex and sexual intercourse just after their 18th birthday (at age 18.11 and at age 18.25 , respectively). Based on median ages reported in 5-year cohorts, the sexual
Fig. 3 Median ages of first masturbation, French kissing, and manual sex

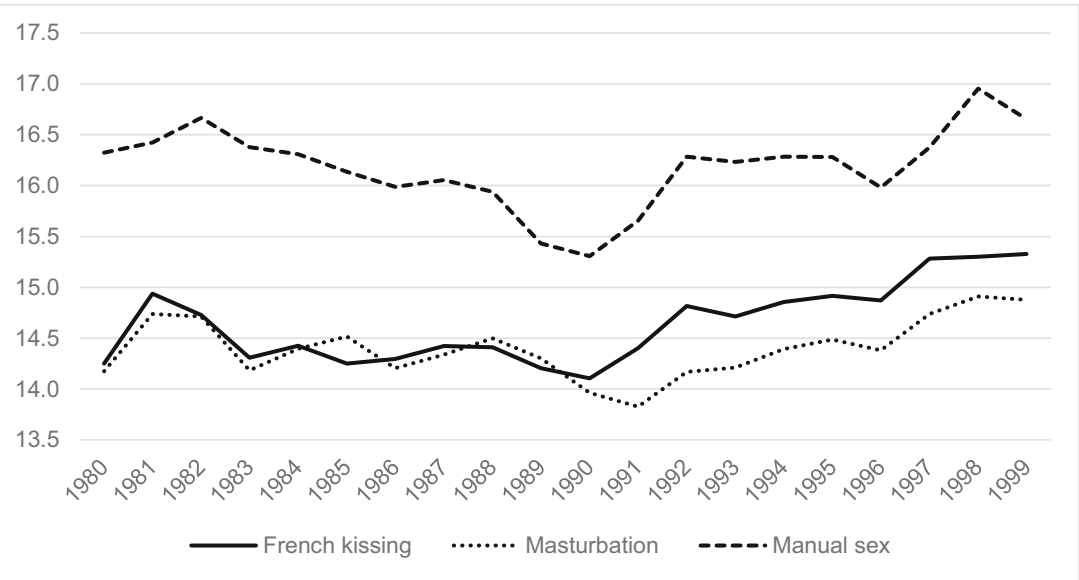


debut ages in the Netherlands seem to be a bit higher than in the United Kingdom (Lewis et al. 2017; Mercer et al. 2013) and quite similar to or a bit younger than the United States (Cavazos-Rehg et al. 2009; Finer 2007). However, none of these studies reported on the ages of different first sexual experiences in the current-generation adolescents with this precision.

The estimates of sexual debut ages for the current generation of Dutch adolescents are considerably higher than a few decades ago: the median age of first sexual intercourse has increased 0.08 ( 1 month) each year. This confirms the findings of recent studies. Ethier et al. (2018) reported on a decrease in sexual activity among 9th and 10th graders between 2005 and 2015. Twenge and Park (2019) found that in recent years, fewer 9th-12th graders engaged in adult activities, which include dating and having sex. Both studies noted that the decline was particularly steep in recent years, i.e., for young people born in the late 1990s. Other studies also showed a decline in the proportion of sexually active 15 year-olds in Scotland and Portugal between 2002 and 2014 (Neville et al. 2017; Reis et al. 2018). Evidence from earlier studies showed mixed results. The proportion of sexually active 15-year-olds remained more or less constant between 2002 and 2010 in 20 European countries (Ramiro et al. 2015). However, both studies on trends in Britain between 1990 and 2012 found a lower median age of first sexual intercourse among the youngest cohorts (Lewis et al. 2017; Mercer et al. 2013).

Our findings raise the question of why the current generation of Dutch youth is waiting longer to have intercourse. To offer some explanations, we investigated a few other changes in the context of this generation that co-occur with this phenomenon. The first area that comes to mind is social media use, since there have been massive changes in recent decades in this regard (Twenge et al. 2018). Social media usage has been suggested as a barrier to entering into offline sexual relationships, for example because online sexual experimentation could replace the desire for offline contact. A recent meta-analysis of Madigan et al. (2018) showed that engagement in "sexting" (i.e., exchanging sexual images) indeed has increased over the years. However, sexting behavior generally correlates with a higher chance of being sexually active, so it does not seem to be a substitute for offline sexual experiences (Ybarra and Mitchell 2014). Another suggested effect of social media usage is that it increases insecurity regarding appearance and sexual performance (Doornwaard et al. 2014; Rodgers et al. 2015). Young people are confronted with images of perfect bodies and sexual interactions and feel pressured to live up to this. Hence, they feel anxious about entering into a sexual relationship. This might explain why the shifts in median ages were more evident for forms of partnered sexual behavior where the body is clearly visible.

The developmental delay, however, is present not only in the area of sexuality, but also in other areas that are generally associated with adulthood, such as drinking, driving, working for pay, marriage, and parenting (Twenge and Park 2019; Sawyer et al. 2018). Thus, the later age of sexual debut appears to be part of a slower path in general. This slower life course has been ascribed to the fact that there is no need for young people to grow up fast these days. Their parents supply them with everything they need (emotional as well as material), and they live long and healthy lives (Twenge and Park 2019).

The present study also has a number of limitations. Although the Cox proportional hazards model offers a good approach for estimating debut ages and investigating trends, the model is not applicable for all forms of sexual behavior and all data sets. The model has a number of fairly strict assumptions, and the results are only easy to communicate as linear shifts if the yearly changes in hazards are small and the age range of the sample is not too large. If the Cox model is not applicable, researchers are left with the other methods for estimating sexual debut ages described in the introduction, or with separate estimates for 1-year cohorts, each with their own limitations.

We do not recommend using the Cox model to estimate the ages of first experiences for young people who are not yet included in the data set (in our data, those born after 2004), since no data can confirm that the proportional hazard change estimated in the included birth years will also continue in years that are not yet included. Caution is warranted in using our proposed method for cohorts in which the majority have not yet had this sexual experience (in our data, those born between 1999 and 2004 for sexual intercourse), since for these younger cohorts the Cox model can only estimate proportional hazard changes at ages younger than the median age, and our approach relies on the assumption that these continue at least until the median age (for details on assumption checks, see Ter Schure 2020). In general, we cannot assume that the linear trend that we found in our data will continue in the future. It is very well possible that the changes that we found will level off. Monitoring the sexual behavior of the new generation of youth, therefore, remains important.

Furthermore, although we offered some explanations for the trends that we found, we are not able to confirm or reject these hypotheses based on our data. We used data from a survey which encompasses many sexual health topics. Because the time needed to complete the survey was limited, we were not able to include questions on other life domains, such as social media use or parenting styles. Moreover, our study design is cross-sectional, which makes conclusions about causal relationships impossible. A longitudinal study that covers many different developmental areas is recommended, if we want to shed light on the processes behind the slowing of adolescents' sexual trajectories.

In sum, young people in the Netherlands have their first experiences of petting, oral sex, and sexual intercourse at a 
later age than youth born in former decennia. By pooling information across birth-year cohorts, Cox regression yields precise and current debut ages. We estimated that Dutch adolescents are currently (2021) approximately 18 years old when they have their first experiences of sexual intercourse or oral sex. This is a positive development, since an early sexual debut is associated with adverse outcomes (Sandfort et al. 2008; Zimmer-Gembeck and Helfand 2008). This median age of first sexual intercourse should not lead to the conclusion that sexuality education can wait. For those who start much earlier, sexuality education is key. However, for those who start at a median age, there is a large time gap between receiving sexuality education and their sexual debut. Hence, continuous sexuality education throughout high school is advisable.

Acknowledgements Fifteen Municipal Health Services (GGDs) made a major contribution to the recruitment of a large database containing national as well as regional data.

Author contributions All authors contributed to the study conception and design. Hanneke de Graaf coordinated the study. Judith ter Schure performed the statistical analysis and participated in the interpretation of the results. Geneviève van Liere participated in data collection. All authors read and approved the final manuscript.

Funding This research was supported by a grant from the Dutch National Institute for Public Health and the Environment (RIVM), the Netherlands (grant number 323846).

\section{Declarations}

Conflict of interest The authors declare that they have no conflict of interest.

Ethical approval The MREC UMC Utrecht confirmed that the Medical Research Involving Human Subjects Act (WMO) does not apply to Sex under the age of 25 , and therefore an official approval of this study by the MREC UMC Utrecht is not required under the WMO (reference number $\mathrm{WAG} / \mathrm{mb} / 16 / 013562$ )

Informed consent For in-school youth, school boards provided active informed consent for participation of their students. In-school students and their parents provided passive informed assent and consent, respectively, where they were able to refuse voluntary participation. Out-ofschool youth ( $\geq 17$ years) provided active informed consent.

\section{References}

Buhi ER, Goodson P (2007) Predictors of adolescent sexual behavior and intention: a theory-guided systematic review. J Adolesc Health 40: $4-21$

BZgA (Federal Centre for Health Education) (2010) Standards for sexuality education in Europe. BZgA, Cologne

Cavazos-Rehg PA, Krauss MJ, Spitznagel EL, Schootman M, Bucholz KK, Peipert JF et al (2009) Age of sexual debut among US adolescents. Contraception 80:158-162
Cha S, Masho SW, Mezuk B (2016) Age of sexual debut and cannabis use in the United States. Subst Use Misuse 51:439-448

De Graaf H, Vanwesenbeeck I, Meijer S, Woertman L, Meeus W (2009) Sexual trajectories during adolescence: relation to demographic characteristics and sexual risk. Arch Sex Behav 38(2):276-282

De Graaf H, Vanwesenbeeck I, Meijer S (2015) Educational differences in adolescents' sexual health: a pervasive phenomenon in a national dutch sample. The J Sex Res 52(7):747-757

De Graaf H, Verbeek M, Van den Borne M, Meijer S (2018) Offline and online sexual risk behavior among youth in the netherlands: findings from "sex under the age of 25". Frontiers in Public Health 6

De Irala J, Osorio A, Ruiz-Canela M, Carlos S, Lopez-del Burgo C (2014) Informing youth about the age of sexual initiation using means or percentages. Health Commun 29:629-633

Doornwaard SM, Bickham DS, Rich M, Vanwesenbeeck I, Van den Eijnden RJJM, Ter Bogt TFM (2014) Sex-related online behaviors and adolescents' body and sexual self-perceptions. Pediatrics 134: $1103-1110$

Epstein M, Furlong M, Kosterman R, Bailey JA, King KM, Vasilenko SA et al (2018) Adolescent age of sexual initiation and subsequent adult health outcomes. Am J Public Health 108:822-828

Ethier KA, Kann L, McManus T (2018) Sexual intercourse among high school students - 29 states and United States overall, 2005-2015. Morb Mortal Wkly Rep 66:1393-1397

Finer LB (2007) Trends in premarital sex in the United States, 1954 2003. Public Health Rep 122:73-78

Golden RL, Furman W, Collibee C (2016) The risks and rewards of sexual debut. Dev Psychol 52:1913-1925

Halpern CT, Haydon AA (2012) Sexual timetables for oral-genital, vaginal, and anal intercourse: sociodemographic comparisons in a nationally representative sample of adolescents. Am J Public Health 102:1221-1228

Haydon AA, Herring AH, Prinstein MJ, Halpern CT (2012) Beyond age of first sex: patterns of emerging sexual behavior in adolescence and young adulthood. J Adolesc Health 50:456-463

Holway GV (2015) Vaginal and oral sex initiation timing: a focus on gender and race/ethnicity. Int J Sex Health 27:351-367

Ihongbe TOA, Cha S, Masho SW (2017) Age of sexual debut and physical dating violence victimization: sex differences among US high school students. J Sch Health 87:200-208

Lara LAS, Abda CHN (2016) Age at time of initial sexual intercourse and health of adolescent girls. J Pediatr Adolesc Gynecol 29:417-423

Lewis R, Tanton C, Mercer CH, Mitchell KR, Palmer M, Macdowall W, Wellings K (2017) Heterosexual practices among young people in Britain: evidence from three national surveys of sexual attitudes and lifestyles. J Adolesc Health 61:694e702

Liu G, Hariri S, Bradley H, Gottlieb SL, Leichliter JS, Markowitz LE (2015) Trends and patterns of sexual behaviors among adolescents and adults aged 14 to 59 years, United States. Sex Transm Dis 42: 20-26

Madigan S, Ly A, Rash CL, Van Ouytsel J, Temple JR (2018) Prevalence of multiple forms of sexting behavior among youth: a systematic review and meta-analysis. JAMA Pediatr 172:327-335

Madkour SA, De Looze M, Maa P, Halpern CT, Farhat T, Ter Bogt TFM et al (2014) Macro-level age norms for the timing of sexual initiation and adolescents' early sexual initiation in 17 European countries. $\mathrm{J}$ Adolesc Health 55:114-121

Mercer CH, Tanton C, Prah P, Erens B, Sonnenberg P, Clifton S et al (2013) Changes in sexual attitudes and lifestyles in Britain through the life course and over time: findings from the National Surveys of Sexual Attitudes and Lifestyles (Natsal). Lancet 382:1781-1794

Neville FG, McEachran J, Aleman-Diaz A, Whitehead R, Cosma A, Currie D, Currie C (2017) Trends in the sexual behaviour of 15year olds in Scotland: 2002-14. Eur J Public Health 27:835-839

Osorio A, Lopez-del Burgo C, Carlos S, De Irala J (2017) The sooner, the worse? Association between earlier age of sexual initiation and 
worse adolescent health and well-being outcomes. Front Psychol 8: 1298

Ramiro K, Windlin B, Reis M, Nic Gabhainn S, Jovic S, Matos MG et al (2015) Gendered trends in early and very early sex and condom use in 20 European countries from 2002 to 2010. Eur J Pub Health 25: $65-68$

Reis M, Ramiro L, Camacho I, Tomé G, De Matos MG (2018) Trends in Portuguese adolescents' sexual behavior from 2002 to 2014: HBSC Portuguese study. Rev Port Sau Pub 36:32-40

Rodgers RF, McLean SA, Paxton SJ (2015) Longitudinal relationships among internalization of the media ideal, peer social comparison, and body dissatisfaction: implications for the tripartite influence model. Dev Psychol 51:706-713

Sandfort TGM, Orr M, Hirsch J, Santelli J (2008) Long-term health correlates of timing of sexual debut: results from a national US study. Am J Public Health 98:155-161

Sawyer SM, Azzopardi PS, Wickremarathne D, Patton GC (2018) The age of adolescence. Lancet Child Adolesc Health 2:223-228

Ter Schure J (2020) Step-by-step assumptions underlying the use of Cox proportional hazards modeling for median sexual debut age estimation in age-censored data with generational trends. https://doi.org/ 10.17605/OSF.IO/XBQ2Z

Tornello SL, Riskind RF, Patterson C (2014) Sexual orientation and sexual and reproductive health among adolescent young women in the United States. J Adolesc Health 54:160-168

Twenge JM, Park H (2019) The decline in adult activities among U.S. adolescents, 1976-2016. Child Dev 90:638-654
Twenge JM, Martin GN, Spitzberg BH (2018) Trends in U.S. adolescents' media use, 1976-2016: the rise of digital media, the decline of TV, and the (near) demise of print. Psychol Pop Media Cult 8:329 345

Vasilenko SE, Kugler KC, Rice CE (2016) Timing of first sexual intercourse and young adult health outcomes. J Adolesc Health 59:291297

Ybarra ML, Mitchell KJ (2014) "Sexting" and its relation to sexual activity and sexual risk behavior in a national survey of adolescents. J Adolesc Health 55:757-764

Young H, Burke L, Gabhainn SN (2018) Sexual intercourse, age of initiation and contraception among adolescents in Ireland: findings from the Health Behaviour in School-aged Children (HBSC) Ireland study. BMC Public Health 18:362

Zaba B, Boerma T, Pisani E, Baptiste N (2002) Estimation of levels and trends in age at first sex from surveys using survival analysis. Chapel Hill, Carolina Population Center

Zaba B, Pisani E, Slaymaker E, Boerma JT (2004) Age at first sex: understanding recent trends in African demographic surveys. Sex Transm Infect 80:ii28-ii35

Zimmer-Gembeck MJ, Helfand M (2008) Ten years of longitudinal research on US adolescent sexual behavior: developmental correlates of sexual intercourse, and the importance of age, gender and ethnic background. Dev Rev 28:153-224

Publisher's note Springer Nature remains neutral with regard to jurisdictional claims in published maps and institutional affiliations. 